\title{
RETRACTED ARTICLE: Relationship of Serum Heart-Type Fatty Acid-Binding Protein Levels and Cerebral Infarction: a Meta-analysis
}

\author{
Bao-Liang Sun • Ming-Feng Yang • Xiao-Yi Yang • Jing-Yi Sun • Da-Wei Li • \\ Zong-Yong Zhang • Lei-Lei Mao • Cun-Dong Fan • Cheng-Bi Zheng • Hong-Li Gao • \\ Feng-Ze Wang • Feng Zhang • Hui Yuan
}

Received: 20 June 2014 / Accepted: 1 September 2014 / Published online: 17 August 2015

(C) Springer Science+Business Media New York 2014

The Publisher and Editor retract this article in accordance with the recommendations of the Committee on Publication Ethics (COPE). After a thorough investigation we have strong reason to believe that the peer review process was compromised.

The original article was published online on September 28, 2014.

B.-L. Sun $(\bowtie) \cdot$ M.-F. Yang $\cdot$ X.-Y. Yang $\cdot$ D.-W. Li · Z.-Y. Zhang •

L.-L. Mao • C.-D. Fan • C.-B. Zheng • H.-L. Gao • F.-Z. Wang •

F. Zhang $\cdot H$. Yuan $(\bowtie)$

Key Lab of Cerebral Microcirculation in Universities

of Shandong, Taishan Medical University, Taian 271000,

People's Republic of China

e-mail: blsun@tsmc.edu.cn

e-mail: yuanhui0314@163.com

\section{B.-L. Sun $\cdot$ H. Yuan}

Department of Neurology, Affiliated Hospital of Taishan

Medical University, NO.2 East Yingsheng Road,

Taian 271000, People's Republic of China

\section{J.-Y. Sun}

Department of Clinical Medicine, Taishan Medical University,

Taian 271000, People's Republic of China

C.-B. Zheng

Library, Taishan Medical University,

Taian 271016, People's Republic of China

\section{H.-L. Gao}

School of Pharmacy, Taishan Medical University,

Taian 271016, People's Republic of China

\section{F.-Z. Wang}

School of Biological Science, Taishan Medical University,

Taian 271016, People's Republic of China 